\title{
PERDAS DE SOLO, MATÉRIA ORGÂNICA E NUTRIENTES POR EROSÃO HÍDRICA EM UMA VERTENTE COBERTA COM DIFERENTES QUANTIDADES DE PALHA DE CANA-DE-AÇÚCAR EM GUARIBA - SP
}

\section{GASPARINO B. SOUSA ${ }^{1}$, MARCÍLIO V. MARTINS FILHO², SAMMY S. R. MATIAS ${ }^{1}$}

RESUMO: A erosão em entressulcos resulta da desagregação causada pelo impacto das gotas de chuva na superfície do solo e pelo transporte superficial das partículas do solo desagregadas, onde se encontram a matéria orgânica e os nutrientes fundamentais para a produção agrícola. $\mathrm{O}$ presente trabalho teve como objetivo avaliar as perdas de solo, matéria orgânica e nutrientes em uma vertente localizada em uma área de Latossolo Vermelho-Amarelo distrófico, cultivada com cana-de-açúcar, que é colhida mecanicamente. As parcelas experimentais foram submetidas à ação de uma chuva simulada com intensidade de $60 \mathrm{~mm} \mathrm{~h}^{-1}$, durante 65 minutos. Foram feitas análises do sedimento erodido para a determinação do volume de solução, das perdas de solo, matéria orgânica e nutrientes. Houve maiores perdas de solo, matéria orgânica e nutrientes nos sedimentos oriundos das parcelas com $0 \%$ e $25 \%$ de cobertura por palha de cana-de-açúcar. Em média, essas perdas foram reduzidas nas parcelas com $75 \%$ e $100 \%$ de cobertura com palha de cana-de-açúcar. Os resultados permitiram concluir que uma cobertura com palha de cana-de-açúcar acima de $50 \%$, da área colhida, reduz a perda de solo e de matéria orgânica, bem como diminui a concentração de nutrientes no sedimento erodido.

PALAVRAS-CHAVE: erosão em entressulcos, chuva simulada, palhada, Latossolo Vermelho.

\section{SOIL, ORGANIC MATTER AND NUTRIENTS LOSSES BY WATER EROSION IN A SLOPE WITH SUGARCANE STRAW, IN GUARIBA, STATE OF SÃO PAULO}

\begin{abstract}
The interril erosion results of the disaggregation caused by the impact of the rainfall on the surface and by the superficial transport of the disaggregated soil particles, where there are the organic matter and the nutrients for the agricultural production. The present work aimed to evaluate the soil, organic matter and nutrients losses of a slope located in an area of distrofic Oxisol, cultivated with sugarcane and submitted to the automated crop. Experimental portions were submitted to the action of a simulated rain of $60 \mathrm{~mm} \mathrm{~h}^{-1}$ intensity, for 65 minutes. Analyses of the erosion sediment were made to determine the solution volume and soil, organic matter and nutrients losses. There were larger soil, organic matter and nutrients losses in the sediment of the portions with $0 \%$ and $25 \%$ of sugarcane straw coverage. On average, the losses were significantly reduced in the portions with $75 \%$ and $100 \%$ of sugarcane straw coverage. The results allowed concluding that the sugarcane straw coverage above $50 \%$, in the crop area, reduces the soil and organic matter loss as well as the concentration of nutrients in the erosion sediment.
\end{abstract}

KEYWORDS: interrill erosion, rainfall simulation, straw, Oxisol.

\footnotetext{
${ }^{1}$ Prof. Dr., Universidade Estadual do Piauí, UESPI, Rua Profa. Joaquina Nogueira de Oliveira, s/n, Aeroporto, Corrente - PI, gasparinobj@ hotmail.com; ymmsa2001@yahoo.com.br.

${ }^{2}$ Prof. Dr., Universidade Estadual Paulista, UNESP, Via de Acesso Prof. Paulo Donato Castelane, s/n, Jaboticabal - SP, mfilho@fcav.unesp.br.

Recebido pelo Conselho Editorial em: 4-11-2010

Aprovado pelo Conselho Editorial em: 9-1-2012
} 


\section{INTRODUÇÃO}

O transporte e as perdas de fertilizantes, pesticidas e resíduos animais ou industriais na enxurrada têm sido objeto de estudo em vários trabalhos experimentais (MARTINS FILHO et al., 2009). Dois problemas prioritários devem merecer investigação: 1) o desenvolvimento de novas técnicas de manejo dos resíduos para uma redução mais efetiva da erosão hídrica, relacionando práticas de produção com a cobertura residual; 2) o desenvolvimento de métodos mais eficientes de uso de fertilizantes, pesticidas e resíduos animais.

O transporte de solo pode ocorrer em terras cultivadas como resultado da interação de processos erosivos a partir do escoamento superficial. A erosão hídrica ou eólica motivam as perdas do solo e podem ainda modificar as propriedades físicas do solo reduzindo a produtividade das culturas. O efeito do transporte de partículas do solo, como uma combinação dos processos erosivos aumenta a variabilidade espacial das produções agrícolas, assim como a do declínio global da capacidade produtiva do solo.

Podem ocorrer reduções nas produtividades das culturas, embora isto dependa do contraste entre propriedades do topo e do subsolo em termos de textura, fertilidade e estágio do processo erosivo (BAKKER et al., 2005).

A água é o elemento-motor dos processos erosivos, atuando no transporte vertical e lateral de materiais em suspensões ou soluções, e nas transformações físicas, químicas e biológicas do solo. Constitui assim um elo comum entre os solos da paisagem, interpolando-os continuamente ao longo dos declives (MARQUES JÚNIOR, 1995).

A remoção das substâncias químicas em solução na água de enxurrada, em áreas agrícolas, tem potencial para ser uma significativa fonte de perdas destas substâncias em sistemas como de cultivo convencional, plantio direto e com cobertura morta. Substâncias químicas podem ser transportadas em solução pelo fluxo superficial e/ou adsorvidas nos sedimentos suspensos no escoamento.

Estudos têm mostrado que sistemas de conservação do solo como plantio direto e cultivo mínimo diminuem a erosão e as perdas de substâncias químicas adsorvidas ao sedimento (BERTOL et al., 2008). Contudo, as perdas de produtos químicos em solução poderão ser consideravelmente elevadas. Isto ocorre porque, em sistemas conservacionistas como os mencionados, há grande utilização de agrotóxicos e herbicidas, que são aplicados e levemente incorporados na superfície do solo (BERTOL et al., 2007).

Com a introdução da colheita mecanizada na cultura de cana-de-açúcar, algumas áreas canavieiras diminuíram o potencial para perdas de produtos químicos transportados em solução no fluxo de enxurrada. Tal afirmação justifica-se pelos seguintes motivos: 1) neste sistema não há despalha pelo fogo, as perdas de solo são reduzidas devido a um menor número de operações de cultivo e, ainda, pela proteção oferecida pela camada de resíduos deixada sobre a superfície após a colheita da cana; e 2) as aplicações de fertilizantes e corretivos ocorrem pela distribuição destes produtos sobre a superfície do solo.

MARTINS FILHO et al. (2009) concluíram que as perdas de nutrientes no material em suspensão da enxurrada são pequenas, quando comparadas com as quantidades de fertilizantes aplicados ao solo. Sendo assim, as perdas de nutrientes em sistemas que eliminam os resíduos culturais, como é o caso no cultivo da cultura de cana-de-açúcar, com a queima da palhada antes da colheita, devem ser reavaliadas, em relação a essas perdas.

Considerando-se o exposto, o presente trabalho teve como objetivos avaliar as perdas de solo, matéria orgânica e nutrientes em uma vertente com diferentes quantidades de cobertura com palha de cana-de-açúcar, em Guariba - SP. 


\section{MATERIAL E MÉTODOS}

A área de estudo localiza-se no nordeste do Estado de São Paulo, no Município de Guariba, na fazenda São Bento, da Usina São Martinho. As coordenadas geográficas locais são $21^{\circ} 19^{\prime}$ de latitude sul e $48^{\circ} 13^{\prime}$ 'de longitude oeste, com altitude média de $640 \mathrm{~m}$ acima do nível do mar. O clima da região, segundo a classificação de Köeppen, é do tipo mesotérmico com inverno seco (Aw), com precipitação média de $1.400 \mathrm{~mm}$, com chuvas concentradas no período de novembro a fevereiro. A vegetação natural era constituída por floresta tropical subcaducifólia .

Os solos das áreas foram classificados como Latossolo Vermelho-Amarelo distrófico (LVAd) (EMBRAPA, 2006), sob cultivo de cana-de-açúcar. Os valores dos principais atributos químicos e físicos são apresentados nas Tabelas 1 e 2, e foram determinados para amostras coletadas no topo, na encosta e no sopé de vertente.

TABELA 1. Atributos químicos do solo na profundidade $0-0,20 \mathrm{~m}$. Soil chemical atributes in $\mathbf{0}$ 0,20m depth.

\begin{tabular}{lccccccccc}
\hline Posição & $\begin{array}{c}\mathrm{MO} \\
\mathrm{gdm}^{-3}\end{array}$ & $\begin{array}{c}\mathrm{P} \\
\mathrm{mg} \mathrm{dm}^{-3}\end{array}$ & $\begin{array}{c}\mathrm{K} \\
-------------\end{array}$ & $\begin{array}{c}\mathrm{Ca} \\
\mathrm{mmol}_{\mathrm{c}} \mathrm{dm}^{-3}------------\end{array}$ & $\mathrm{H}$ & $\mathrm{H}$ \\
\hline Topo & 24,0 & 36 & 1 & 31 & 9,0 & 37 & 41 & 78 & 52 \\
\hline Encosta & 29,4 & 33,2 & 1,5 & 30,5 & 9,0 & 33,6 & 41 & 75 & 54 \\
\hline Sopé & 19,4 & 21,6 & 1,2 & 18,4 & 5,2 & 36,2 & 24,8 & 61 & 40,5 \\
\hline
\end{tabular}

TABELA 2. Atributos físicos do solo na profundidade 0-0,20m. Physical soil attributes in 0-0,20 m depth.

\begin{tabular}{lccccc}
\hline Posição & Argila & Silte & AT & AG & AF \\
& ------ & $\mathrm{g} \mathrm{kg}^{-1}$ & \\
\hline Topo & 300 & 60 & 640 & 380 & 260 \\
\hline Encosta & 260 & 70 & 670 & 340 & 330 \\
\hline Sopé & 340 & 80 & 580 & 480 & 100 \\
\hline
\end{tabular}

AT - Areia total; AG - Areia grossa, e AF - Areia fina

Cumpre ressaltar que a área apresenta um histórico de mais de 20 anos consecutivos com cultivo de cana-de-açúcar. Há cinco anos, foi adotado o plantio direto em substituição ao preparo do solo convencional, aração com duas gradagens, então utilizado. Deste modo, a cobertura vegetal na área, na época da realização deste trabalho, era constituída por resíduos de palha de cana-de-açúcar, os quais foram mantidos sobre a superfície após a colheita mecânica da cultura.

Parcelas experimentais com as dimensões de $0,50 \mathrm{~m}$ de largura por 1,0 $\mathrm{m}$ de comprimento $\left(0,5 \mathrm{~m}^{2}\right)$ e delimitadas com chapas metálicas em suas laterais e parte superior, e na extremidade inferior, por uma calha convergente para uma saída de $0,10 \mathrm{~m}$ de diâmetro, foram utilizadas para medir o fluxo de enxurrada do processo de erosão em entressulcos.

O delineamento experimental foi o inteiramente casualizado (DIC), num esquema fatorial, com: três posições na vertente (topo com declividade $0,025 \mathrm{~m} \mathrm{~m}^{-1}$, meia-encosta com declividade $0,05 \mathrm{~m} \mathrm{~m}^{-1}$ e sopé com $0,07 \mathrm{~m} \mathrm{~m}^{-1}$ ), 5 tratamentos com diferentes quantidades de palha de cana-de-açúcar e quatro repetições, totalizando 60 parcelas. Nos tratamentos (parcelas), a palha foi rearranjada manualmente na superfície do solo, de modo que se obtiveram $0 \%\left(\mathrm{CS}_{0}\right), 25 \%\left(\mathrm{CS}_{25}\right)$, $50 \%\left(\mathrm{CS}_{50}\right), 75 \%\left(\mathrm{CS}_{75}\right)$ e $100 \%\left(\mathrm{CS}_{100}\right)$ de área de solo coberta com palha de cana-de-açúcar. As parcelas--testemunha não receberam cobertura de palha de cana-de-açúcar $\left(\mathrm{CS}_{0}\right)$. Os tratamentos cobertura com palha foram diferentes para cada parcela, considerando $1,4 \mathrm{~kg} \mathrm{~m}^{-2}$ de palha como $100 \%$ de cobertura. Para chegar a esse valor, pesaram-se várias amostras de $1 \mathrm{~m}^{2}$ de palha na área após a colheita, e determinou-se a média das amostras. A aplicação da palha foi realizada 2 horas antes da realização de chuva simulada e das avaliações de erosão em entressulcos. 
As parcelas experimentais foram submetidas a chuvas simuladas com intensidades médias de $60 \mathrm{~mm} \mathrm{~h}^{-1}$, durante 65 minutos. Utilizou-se um simulador de chuva de hastes rotativas do tipo Swanson, com bicos Veejet 80-100, previamente calibrado e nivelado no terreno. Trinta e três pluviômetros, alinhados no sentido do declive, na área de ação do simulador de chuvas, como descrito por MARTINS FILHO et al. (2009), foram utilizados para determinar as intensidades das precipitações produzidas pelo simulador, nas parcelas experimentais.

Para as medidas de vazões dos escoamentos superficiais e das concentrações de sedimentos, foram coletadas amostras no quinto minuto após o início da enxurrada e, a partir daí, a cada cinco minutos. Amostras foram coletadas em recipientes de plástico com capacidade de 1 L, cronometrando-se o tempo de coleta. Logo após as coletas, os recipientes foram fechados e conduzidos ao laboratório, para a quantificação da concentração de sedimentos e volume de solução, e consequente determinação das taxas de perdas de solo e água.

Os volumes de solução coletados foram pesados, em balança com resolução de 0,01 g, e, em seguida, as amostras deixadas em repouso por 24 horas, para a deposição dos sedimentos. Após o período de repouso de 24 horas, o sobrenadante foi filtrado e submetido à análise direta de determinação de P, K, Ca e Mg, seguindo método da EMBRAPA (1979). Já o material decantado foi levado à estufa a $60^{\circ} \mathrm{C}$ até secagem completa. Após secagem, as amostras foram pesadas, determinando-se o peso do sedimento de cada uma. A concentração de sedimentos foi obtida considerando-se o volume da solução, a densidade da água e do sedimento na solução. Cada um dos volumes de solução obtidos foram divididos pelo tempo de coleta, obtendo-se a vazão de enxurrada em cada intervalo de coleta.

Uma vez quantificado o sedimento erodido em $\mathrm{kg} \mathrm{s}^{-1}$ e a vazão em $\mathrm{m}^{3} \mathrm{~s}^{-1}$, para cada amostragem realizada, a taxa de erosão (A) e a de enxurrada (R) foram determinadas dividindo-se cada um dos valores obtidos pela área de cada parcela. A taxa de erosão global, a intensidade média das chuvas aplicadas, a taxa de enxurrada e a declividade média das parcelas sem cobertura vegetal foram utilizadas para a determinação da erodibilidade, fator K. Já os mesmos dados relativos às parcelas com cobertura vegetal foram utilizados na determinação do subfator cobertura vegetal e manejo do solo. Amostras deformadas foram coletadas nas bordaduras das parcelas entressulcos, com o auxílio de um trado, no intervalo de profundidade de $0-0,15 \mathrm{~m}$, para a determinação da umidade do solo. A umidade do solo foi determinada gravimetricamente, a partir de amostras coletadas no campo. No laboratório, as amostras foram pesadas e secas em estufa sob circulação forçada a $105{ }^{\circ} \mathrm{C}$, por 24 horas, e pesadas novamente. A umidade gravimétrica foi expressa em $\mathrm{g} \mathrm{g}^{-1}$ e posteriormente convertida $\mathrm{em}^{3} \mathrm{~m}^{-3}$, utilizando-se da densidade do solo.

Para a caracterização química do solo e do sedimento erodido, o cálcio, o magnésio, o potássio e o fósforo foram extraídos pelo método da resina trocadora de íons de acordo com a metodologia de RAIJ et al. (2001). O carbono orgânico $(\mathrm{C} \%)$ foi determinado seguindo metodologia da EMBRAPA (1979). O pH foi determinado potenciometricamente em solução de $\mathrm{CaCl}_{2} 0,01 \mathrm{M}$.

Os resultados foram submetidos à análise da variância, segundo o delineamento fatorial, sendo que, para as comparações múltiplas das médias, utilizou-se o teste de Duncan, a 5\%. Diferenças com nível mínimo de significância inferior a 5\% foram consideradas significativas. Todos os resultados das análises estatísticas foram obtidos com o programa Statistica. As taxas de desagregação do solo em entressulcos (Di, $\mathrm{kg} \mathrm{m}^{-2} \mathrm{~s}^{-1}$ ) foram determinadas conforme eq.(1):

$$
\mathrm{Di}=\frac{m s}{t A}
$$

em que,

ms - massa de solo desagregado, $\mathrm{kg}$;

$\mathrm{t}$ - tempo de coleta, $\mathrm{s}$, e

A - área da parcela, $\mathrm{m}^{2}$. 
As perdas totais de solo foram determinadas pela eq.(2):

$$
\mathrm{PS}=\frac{\sum_{i=1}^{n}\left(\mathrm{Qi} \mathrm{Ci}^{\mathrm{n}}\right)}{\mathrm{A}}
$$

em que,

PS - perda total de solo em entressulcos, $\mathrm{kg} \mathrm{m}^{-2}$;

$\mathrm{Q}$ - vazão, $\mathrm{L} \mathrm{s}^{-1}$;

$\mathrm{C}$ - concentração de sedimentos, $\mathrm{kg} \mathrm{L}^{-1}$;

$\mathrm{t}$ - intervalo entre as coletas, $300 \mathrm{~s}$;

A - área da parcela, $\mathrm{m}^{2}$, e

$\mathrm{n}$ - número total de amostras coletadas.

\section{RESULTADOS E DISCUSSÃO}

A taxa de erosão em entressulcos, mostrada na Tabela 3, apresenta valores significativos, estatisticamente diferentes entre as posições da vertente topo, encosta e sopé, nos percentuais de cobertura $0,0 \%$ e $25 \%$. Isto pode ser explicado pela diferença na declividade, $0,002 \mathrm{~m} \mathrm{~m}^{-1}$, $0,005 \mathrm{~m} \mathrm{~m}^{-1}$ e $0,007 \mathrm{~m} \mathrm{~m}^{-1}$ para topo, encosta e sopé, respectivamente. Os valores mostrados na Tabela 3 comprovam o efeito favorável que a palhada de cana-de-açúcar tem no controle da erosão do solo, o que também foi verificado por CASSOL \& LIMA (2003) e MARTINS FILHO et al. (2009).

TABELA 3. Taxa de erosão em entressulcos. Interrill erosion rate in.

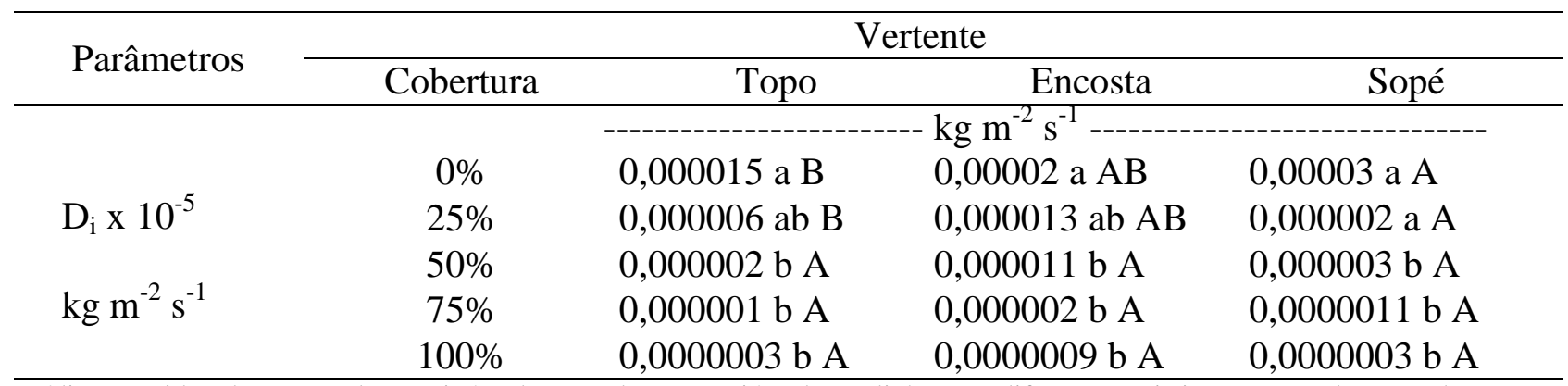

Médias seguidas de mesma letra minúscula na coluna e maiúscula na linha não diferem estatisticamente, pelo teste de Duncan $(\mathrm{p}<0,05)$, para tipos de cobertura e superfície geomórfica.

Pode ser observado que houve diferença estatística significativa para os valores médios de concentração de sedimento entre os percentuais de cobertura $0,0 \%, 25 \%$ e $50 \%$ (Tabela 4). Entre os demais valores $(75 \%$ e $100 \%$ ), não houve diferença estatística significativa. Observa-se que as concentrações de sedimento diminuem quando aumenta o percentual de área ocupada com palha de cana-de-açúcar, o que ocorreu devido ao aumento da quantidade de palha sobre o solo. Pois, quanto mais coberto o solo, maior a proteção contra o impacto direto das gotas de chuva. Esses valores estão de acordo com os resultados obtidos por MARTINS FILHO et al. (2009). Possivelmente, a palha de cana-de-açúcar também ajudou a reduzir a enxurrada. As concentrações de matéria orgânica tiveram valores diferentes em todos os percentuais de cobertura, mas, mesmo assim, observou-se seu decréscimo quando aumentou o percentual de cobertura do solo.

As diferenças dos valores médios de concentração dos nutrientes nos sedimentos transportados pela enxurrada, para cada nível de cobertura por palha sobre a superfície do solo, estão apresentadas na Tabela 4. Verificou-se decréscimo das concentrações de nutrientes, quando a porcentagem de cobertura por palha de cana-de-açúcar foi superior a 50\%, sendo bastante expressiva essa redução na cobertura de $100 \%$. Isto ocorreu em consequência da não desagregação do solo pelo impacto direto das gotas da chuva, a exemplo do verificado por BERTOL et al. (2010) para palhadas de milho e trigo mantidas em contato direto com a superfície do solo. 
As concentrações de $\mathrm{P}, \mathrm{Ca}$ e $\mathrm{Mg}$ nos sedimentos transportados foram maiores na cobertura do solo sem palha de cana-de-açúcar, respectivamente 1,$11 ; 1,09$ e 1,15 vezes (Tabela 4), o que concorda com os trabalhos de SCHICK et al. (2000) e BERTOL et al. (2007) ao estudarem perdas de nutrientes em erosão hídrica no cultivo de soja.

TABELA 4. Concentrações médias de (C), MO, P, K, Ca e Mg nos sedimentos transportados pela enxurrada. Medium concentrations of $\mathrm{C}, \mathrm{MO}, \mathrm{P}, \mathrm{K}, \mathrm{Ca}$ and $\mathrm{Mg}$ in the sediments transported by the heavy rain.

\begin{tabular}{|c|c|c|c|c|}
\hline \multirow{2}{*}{ Parâmetros } & \multicolumn{4}{|c|}{ Vertente } \\
\hline & Cobertura & Topo & Encosta & Sopé \\
\hline \multirow{5}{*}{$\begin{array}{c}\mathrm{C} \\
\mathrm{g} \mathrm{L}^{-1}\end{array}$} & $0 \%$ & 3,30 a $A$ & 3,36 a $A$ & 3,93 a $A$ \\
\hline & $25 \%$ & $1,31 \mathrm{~b} \mathrm{~A}$ & $2,47 \mathrm{ab} A$ & $2,92 \mathrm{ab} A$ \\
\hline & $50 \%$ & $0,57 \mathrm{~b} \mathrm{~B}$ & 3,03 a $A$ & $1,05 \mathrm{bc} \mathrm{B}$ \\
\hline & $75 \%$ & $0,55 \mathrm{~b} \mathrm{~A}$ & $0,79 \mathrm{~b} \mathrm{~A}$ & $0,28 \mathrm{c} \mathrm{A}$ \\
\hline & $100 \%$ & $0,17 \mathrm{~b} \mathrm{~A}$ & $0,48 \mathrm{~b} \mathrm{~A}$ & $0,08 \subset \mathrm{A}$ \\
\hline $\mathrm{MO}$ & $0 \%$ & 47,25 a $\mathrm{A}$ & 34,24 a $\mathrm{A}$ & 42,75 a $\mathrm{A}$ \\
\hline \multirow[t]{4}{*}{$\mathrm{g} \mathrm{kg}^{-1}$} & $25 \%$ & $33,22 \mathrm{ab} \mathrm{A}$ & 31,75 a $\mathrm{A}$ & $17,75 \mathrm{ab} A$ \\
\hline & $50 \%$ & $33,25 \mathrm{ab} A$ & 0,00 a B & $22,00 \mathrm{ab} A B$ \\
\hline & $75 \%$ & 24,00 aab A & 12,00 a $\mathrm{A}$ & $11,50 \mathrm{~b} \mathrm{~A}$ \\
\hline & $100 \%$ & $27,25 \mathrm{aA}$ & 22,00 a A & $0,00 \mathrm{~b} \mathrm{~A}$ \\
\hline \multirow{5}{*}{$\begin{array}{c}\mathrm{P} \\
\mathrm{mg} \mathrm{dm}\end{array}$} & $0 \%$ & 72,75 a $\mathrm{A}$ & 70,25 a $\mathrm{A}$ & 47,50 a $\mathrm{A}$ \\
\hline & $25 \%$ & 66,75 a $A$ & $50,75 \mathrm{ab} A B$ & 14,75 a B \\
\hline & $50 \%$ & 65,75 a A & $19,00 \mathrm{ab} A$ & 27,75 a $\mathrm{A}$ \\
\hline & $75 \%$ & 50,25 a $\mathrm{A}$ & $19,00 \mathrm{ab} A$ & 22,25 a A \\
\hline & $100 \%$ & $0,00 \mathrm{~b} \mathrm{~A}$ & $15,25 \mathrm{~b} \mathrm{~A}$ & 26,00 a $\mathrm{A}$ \\
\hline \multirow{5}{*}{$\underset{\mathrm{mmol}_{\mathrm{c}} \mathrm{dm}^{-3}}{\mathrm{~K}}$} & $0 \%$ & $2,92 \mathrm{ab} \mathrm{B}$ & 6,10 a $A$ & 2,55 a B \\
\hline & $25 \%$ & 3,20 a $A$ & $4,42 \mathrm{ab} A$ & 1,37 a $\mathrm{A}$ \\
\hline & $50 \%$ & 3,12 a $A$ & $1,55 \mathrm{~b} \mathrm{~A}$ & 1,35 a $\mathrm{A}$ \\
\hline & $75 \%$ & $1,85 \mathrm{~b} \mathrm{~A}$ & $1,25 \mathrm{~b} \mathrm{~A}$ & 1,50 a $\mathrm{A}$ \\
\hline & $100 \%$ & 0,00 c A & $1,40 \mathrm{~b} \mathrm{~A}$ & 1,52 a $A$ \\
\hline \multirow{5}{*}{$\begin{array}{c}\mathrm{Ca} \\
\mathrm{mmol}_{\mathrm{c}} \mathrm{dm}^{-3}\end{array}$} & $0 \%$ & 56,72 a $\mathrm{A}$ & 43,28 a $\mathrm{A}$ & 46,65 a $A$ \\
\hline & $25 \%$ & 51,92 a $A$ & $38,38 \mathrm{ab} \mathrm{A}$ & $0,00 \mathrm{~b} \mathrm{~B}$ \\
\hline & $50 \%$ & $0,00 \mathrm{~b} \mathrm{~A}$ & $0,00 \mathrm{~b} \mathrm{~A}$ & $12,45 \mathrm{~b} \mathrm{~A}$ \\
\hline & $75 \%$ & $4,25 \mathrm{~b} \mathrm{~A}$ & $0,00 \mathrm{~b} \mathrm{~A}$ & $11,18 \mathrm{~b} \mathrm{~A}$ \\
\hline & $100 \%$ & $0,00 \mathrm{~b} \mathrm{~A}$ & $0,00 \mathrm{~b} \mathrm{~A}$ & $10,00 \mathrm{~b} \mathrm{~A}$ \\
\hline \multirow{5}{*}{$\begin{array}{c}\mathrm{Mg} \\
\mathrm{mmol}_{\mathrm{c}} \mathrm{dm}^{-3}\end{array}$} & $0 \%$ & 22,67 a $A$ & 16,77 a $\mathrm{A}$ & 19,12 a $A$ \\
\hline & $25 \%$ & 19,27 a $A$ & 15,65 a $\mathrm{A}$ & $0,00 \mathrm{~b} \mathrm{~B}$ \\
\hline & $50 \%$ & $0,00 \mathrm{~b} \mathrm{~A}$ & $0,00 \mathrm{~b} \mathrm{~A}$ & $5,32 \mathrm{~b} \mathrm{~A}$ \\
\hline & $75 \%$ & $3,75 \mathrm{~b} \mathrm{~A}$ & $0,00 \mathrm{~b} \mathrm{~A}$ & $4,30 \mathrm{~b} \mathrm{~A}$ \\
\hline & $100 \%$ & $0,00 \mathrm{~b} \mathrm{~A}$ & $0,00 \mathrm{~b} \mathrm{~A}$ & $3,75 \mathrm{~b} \mathrm{~A}$ \\
\hline
\end{tabular}

Médias seguidas de mesma letra minúscula na coluna e maiúscula na linha não diferem estatisticamente, pelo teste de Duncan $(\mathrm{p}<0,05)$, para tipos de cobertura e superfície geomórfica.

No caso dos percentuais de cobertura do solo com palhada de cana-de-açúcar as concentrações de $\mathrm{P}$, no topo e sopé, $\mathrm{K}$ no sopé, Ca no sopé e Mg no sopé mantiveram-se, em média, iguais para os percentuais de $25 \%, 50 \%$ e $75 \%$ (Tabela 4). As menores concentrações para $\mathrm{P}, \mathrm{K}, \mathrm{Ca}$ e Mg foram observadas na camada de cobertura do solo de $100 \%$. As maiores concentrações de P, $\mathrm{Ca}$ e $\mathrm{Mg}$, nos sedimentos das parcelas sem cobertura, podem ser atribuídas à maior concentração desses nutrientes na superfície do solo, de acordo com resultados obtidos por IZIDORO et al. (2005), ao analisar perdas de nutrientes. Os efeitos da cobertura do solo, na concentração de nutrientes, para as parcelas com cobertura $0 \%$ e $25 \%$ com palha de cana-de-açúcar em contato 
direto com a superfície do solo, que constam na Tabela 4, estão de acordo com os resultados de BEZERRA \& CANTALICE (2006), que verificaram maior proteção contra o impacto direto das gotas de chuva, quando se aumentou a quantidade de palha na superfície do solo. Como consequência dessa proteção, há menor selamento da camada superficial do solo e maior infiltração da água no solo. Além disso, os resíduos na superfície do solo reduzem a velocidade do escoamento superficial.

Resultados diferentes foram observados na concentração de $\mathrm{K}$, que ao contrário do $\mathrm{P}, \mathrm{Ca}$ e $\mathrm{Mg}$, os valores de concentração foram maiores nas camadas com $25 \%$, e $50 \%$, na posição topo, e $25 \%$ na encosta (Tabela 4). Isto é explicado pela maior concentração desse elemento na palha de cana-de-açúcar e menor perda de solo pela proteção da cobertura.

Nas superfícies de topo com declividade de $0,02 \mathrm{~m} \mathrm{~m}^{-1}$, encosta declividade $0,05 \mathrm{~m} \mathrm{~m}^{-1}$ e sopé com declividade de $0,07 \mathrm{~m} \mathrm{~m}^{-1}$ (Tabela 4), houve interferência significativa nas concentrações de nutrientes para as três superfícies, principalmente para $\mathrm{P}$ e $\mathrm{K}$ nas coberturas de $0 \%$ e $25 \%$. Isto pode ser explicado pela diferença de relevo entre as superfícies, que ocasiona maior velocidade de escoamento superficial e consequentemente maior transporte de sedimento.

TABELA 5. Taxa de enriquecimento do sedimento (ER), matéria orgânica e nutrientes. Ratios of enrichment of the sediment (ER), organic matter and nutrients.

\begin{tabular}{|c|c|c|c|c|}
\hline \multirow{2}{*}{ Parâmetros } & \multicolumn{4}{|c|}{ Vertente } \\
\hline & Cobertura & Topo & Encosta & Sopé \\
\hline \multirow{5}{*}{ Matéria orgânica } & $0 \%$ & 2,17 a $\mathrm{A}$ & 1,16 a $A$ & 2,20 a $\mathrm{A}$ \\
\hline & $25 \%$ & 1,50 a $\mathrm{A}$ & 1,08 a $\mathrm{A}$ & $0,91 \mathrm{ab} \mathrm{A}$ \\
\hline & $50 \%$ & 1,37 a $\mathrm{A}$ & 0,00 a $A$ & $1,13 \mathrm{ab} \mathrm{A}$ \\
\hline & $75 \%$ & 1,01 a $\mathrm{A}$ & 0,41 a $\mathrm{A}$ & $0,59 \mathrm{~b} \mathrm{~A}$ \\
\hline & $100 \%$ & $0,39 \mathrm{~b} \mathrm{~A}$ & 0,75 a $\mathrm{A}$ & $0,00 \mathrm{~b} \mathrm{~A}$ \\
\hline \multirow{5}{*}{ Fósforo } & $0 \%$ & 0,57 a B & 2,12 a $A$ & 2,20 a $\mathrm{A}$ \\
\hline & $25 \%$ & 0,23 a $A$ & $1,53 \mathrm{ab} A$ & $0,68 \mathrm{~b} \mathrm{~A}$ \\
\hline & $50 \%$ & 0,09 a $\mathrm{A}$ & $0,57 \mathrm{~b} \mathrm{~A}$ & 1,28 a A \\
\hline & $75 \%$ & 0,04 a A & $0,57 \mathrm{ab} A$ & 1,03 a $\mathrm{A}$ \\
\hline & $100 \%$ & 0,00 a $\mathrm{A}$ & 0,46 b A & 1,20 a A \\
\hline \multirow{5}{*}{ Potássio } & $0 \%$ & 2,34 a A & 3,98 a $A$ & 2,21 a $A$ \\
\hline & $25 \%$ & 2,42 a A & $2,89 \mathrm{ab} A$ & 1,19 a $\mathrm{A}$ \\
\hline & $50 \%$ & 1,96 a $\mathrm{A}$ & $1,01 \mathrm{~b} \mathrm{~A}$ & 1,17 a $\mathrm{A}$ \\
\hline & $75 \%$ & 2,36 a $A$ & $0,81 \mathrm{~b} \mathrm{~A}$ & 1,30 a $A$ \\
\hline & $100 \%$ & 0,61 a A & $0,91 \mathrm{~b} \mathrm{~A}$ & 1,32 a $\mathrm{A}$ \\
\hline \multirow{5}{*}{ Cálcio } & $0 \%$ & 2,09 a A & $1,41 \mathrm{a} \mathrm{A}$ & 2,53 a $A$ \\
\hline & $25 \%$ & $1,32 \mathrm{ab} A$ & $1,25 \mathrm{ab} \mathrm{AB}$ & $0,00 \mathrm{~b} \mathrm{~B}$ \\
\hline & $50 \%$ & 0,43 b A & $0,00 \mathrm{~b} \mathrm{~A}$ & $0,67 \mathrm{~b} A$ \\
\hline & $75 \%$ & $0,51 \mathrm{~b} \mathrm{~A}$ & $0,00 \mathrm{~b} \mathrm{~A}$ & $0,60 \mathrm{bA}$ \\
\hline & $100 \%$ & $0,00 \mathrm{~b} \mathrm{~A}$ & $0,00 \mathrm{~b} \mathrm{~A}$ & $0,63 \mathrm{~b} \mathrm{~A}$ \\
\hline \multirow{5}{*}{ Magnésio } & $0 \%$ & 2,94 a A & 1,86 a $\mathrm{A}$ & 3,67 a $B$ \\
\hline & $25 \%$ & $1,67 \mathrm{ab} A$ & $1,73 \mathrm{ab} A$ & $0,00 \mathrm{~b} \mathrm{~B}$ \\
\hline & $50 \%$ & $0,63 \mathrm{~b} \mathrm{~A}$ & $0,00 \mathrm{~b} A$ & $1,02 \mathrm{~b} \mathrm{~A}$ \\
\hline & $75 \%$ & 0,69 b A & $0,00 \mathrm{~b} \mathrm{~A}$ & $0,82 \mathrm{~b} \mathrm{~A}$ \\
\hline & $100 \%$ & $0,00 \mathrm{~b} \mathrm{~A}$ & $0,00 \mathrm{~b} \mathrm{~A}$ & $0,92 \mathrm{~b} \mathrm{~A}$ \\
\hline
\end{tabular}

Médias seguidas de mesma letra minúscula na coluna e maiúscula na linha não diferem estatisticamente, pelo teste de Duncan $(\mathrm{p}<0,05)$, para tipos de cobertura e superfície geomórfica.

As taxas de enriquecimento do sedimento erodido (ER), matéria orgânica (MO) e nutrientes (P, K, Ca e Mg) são apresentadas na Tabela 5. A ER é a relação entre a concentração de MO e/ou 
nutrientes no sedimento erodido (Tabela 5) e no solo original (Tabela 1). Quando ER é maior que 1, significa que o sedimento se encontra enriquecido por matéria orgânica ou nutrientes do solo MARTINS FILHO et al. (2009). Os dados da Tabela 5 mostram que houve enriquecimento da matéria orgânica em todas as superfícies geomórficas, com maiores valores significativos onde o solo tinha $0 \%$ de cobertura. Os menores valores de enriquecimento foram nas coberturas de $100 \%$ e $75 \%$. Desta mesma forma, ocorreu para os nutrientes $\mathrm{P}, \mathrm{Ca}$ e $\mathrm{Mg}$. Para o $\mathrm{K}$, o ER foi maior que 1 em todos os percentuais de cobertura e superfícies, com exceção do percentual de $100 \%$ nas superfícies de topo e encosta. Esses valores estão de acordo com BERTOL et al. (2008) e MARTINS FILHO et al. (2009), que confirmaram o aumento da ER conforme diminui o percentual de cobertura do solo.

Os dados demonstram que as coberturas de $100 \%$ e $75 \%$ do solo com palha de cana-de-açúcar se mostram eficientes, apresentando valores da ER abaixo de 1. Ocorreram algumas exceções para os nutrientes $\mathrm{P}$ e $\mathrm{K}$ na superfície de topo e sopé. Esta ocorrência pode ser em função do maior percentual de declive e área de deposição no sopé.

Estudando ER num Latossolo Vermelho (LV), em uma encosta com declividade de $0,08 \mathrm{~m} \mathrm{~m}^{-1}$, sob cultivo de cana-de-açúcar, despalhada com uso do fogo, IZIDORO et al. (2005) determinaram, com o solo descoberto, taxas de enriquecimento por MO, P, K, Ca e Mg na ordem de 1,$62 ; 3,87 ; 0,73 ; 1,27$ e 1,24 , respectivamente. Observando a Tabela 5 do presente trabalho, as taxas de ER de maiores valores foram 2,21 (MO); 2,20(P); 2,53 (Ca) e 3,67 (Mg) no sopé e 3,98 (K) na encosta, com $0,07 \mathrm{~m} \mathrm{~m}^{-1}$ e $0,05 \mathrm{~m} \mathrm{~m}^{-1}$ de declividade, respectivamente. As diferenças entre os valores das taxas de ER apresentados acima e os valores encontrados por IZIDORO et al. (2005), provavelmente, são devidas às diferenças de topossequências, sendo o sopé da área de deposição.

Para os resultados apresentados, não ocorreu enriquecimento significativo do sedimento para as coberturas de solo de $75 \%$ e $100 \%$, o que demonstra a eficiência da palha de cana-de-açúcar na redução da concentração de material orgânico e nutriente no solo erodido. Esses resultados estão de acordo com os obtidos por MARTINS FILHO et al. (2009) ao trabalharem com perdas de solo e nutrientes em um Argissolo com resíduos vegetais.

A erosão do solo (A) diminuiu com o aumento da percentagem de cobertura oferecida ao solo com palha de cana $(0,0 \% ; 25 \% ; 50 \% ; 75 \%$ e $100 \%)$ (Tabela 6$)$. Tal aspecto reforça o efeito favorável que a palhada tem no controle da erosão do solo, o que também foi verificado por CANTALICE et al. (2009) e MARTINS FILHO et al. (2009).

As diferenças significativas dos valores médios de perda por erosão para cada percentual de cobertura do solo por palha na superfície estão apresentadas na Tabela 6. Houve diferença estatística significativa para perdas de solo (A) nas posições de topo, encosta e sopé, notadamente nos percentuais de cobertura com $0,0 \%$ e $25 \%$, em relação às outras coberturas. Verifica-se um decréscimo importante nos valores à medida que aumentam os percentuais de cobertura. Para a superfície de encosta, houve diferença das coberturas com $0,0 \%$ e $0,25 \%$ para $50 \% ; 75 \%$ e $100 \%$, em que a cobertura de $0,0 \%$ teve perdas de $0,0636 \mathrm{t} \mathrm{ha}^{-1}$, enquanto a cobertura de $75 \%$ e $100 \%$ apresentaram perdas de 0,0332; 0,0061 e 0,0027 $\mathrm{t} \mathrm{ha}^{-1}$, respectivamente. A exemplo do verificado por CANTALICE et al. (2009) em solo coberto com palhada de milho e trigo, a redução da erosão pode ter ocorrido pelo controle do escoamento.

$\mathrm{Na}$ matéria orgânica $(\mathrm{MO})$, as perdas foram significativas em relação às coberturas e às superfícies da vertente. Foi verificada diferença de perda entre a cobertura de 0,0\% e 100\% acima de $95 \%$, tanto na posição de topo como nas demais. Essas perdas também ocorreram de forma decrescente, ou seja, à medida que aumentou a quantidade de palhada na cobertura, a perda de matéria orgânica diminuiu. Resultados semelhantes foram encontrados por FREITAS et al. (2008) ao verificarem que houve redução na perda de $\mathrm{MO}$ em solos cobertos com palhada de feijão-guandu e batata-doce. 
No fósforo (P), houve diferença estatística significativa nos percentuais de $0,0 \%$ e $25 \%$, em relação às demais coberturas, tanto no topo quanto na encosta e no sopé.

TABELA 6. Perdas de solo por erosão (A), matéria orgânica e nutrientes. Soil losses for erosion (A), organic matter and nutrients in function of the \% of covering in the soil.

\begin{tabular}{|c|c|c|c|c|}
\hline \multirow{2}{*}{ Parâmetros } & \multicolumn{4}{|c|}{ Superficies Geomórficas } \\
\hline & Cob & Topo (I) & Encosta (II) & Sopé (III) \\
\hline \multirow{5}{*}{$\begin{array}{l}\text { A } \\
\mathrm{t}^{\mathrm{ha}^{-1}}\end{array}$} & $0 \%$ & 0,0460 a B & 0,0636 a $\mathrm{AB}$ & 0,0959 a $\mathrm{A}$ \\
\hline & $25 \%$ & $0,0186 \mathrm{ab} B$ & $0,0393 \mathrm{ab} \mathrm{AB}$ & 0,0660 a $\mathrm{A}$ \\
\hline & $50 \%$ & 0,0086 b A & $0,0332 \mathrm{ab} A$ & 0,0097 a A \\
\hline & $75 \%$ & 0,0033 b A & $0,0061 \mathrm{~b} \mathrm{~A}$ & 0,0033 b A \\
\hline & $100 \%$ & $0,0010 \mathrm{~b} \mathrm{~A}$ & $0,0027 \mathrm{~b} \mathrm{~A}$ & $0,0011 \mathrm{~b} \mathrm{~A}$ \\
\hline \multirow{5}{*}{$\begin{array}{c}\mathrm{MO} \\
\mathrm{kg} \mathrm{ha}^{-1}\end{array}$} & $0 \%$ & 216,30 a B & 169,67 a B & 391,42 a $A$ \\
\hline & $25 \%$ & $67,16 \mathrm{~b} \mathrm{~A}$ & $83,29 \mathrm{ab} \mathrm{A}$ & 83,47 b A \\
\hline & $50 \%$ & $32,81 \mathrm{~b} \mathrm{~A}$ & $0,00 \mathrm{~b} \mathrm{~A}$ & 13,88 b A \\
\hline & $75 \%$ & 8,39 b A & 3,06 b A & $2,71 \mathrm{~b} \mathrm{~A}$ \\
\hline & $100 \%$ & $4,06 \mathrm{~b} \mathrm{~A}$ & $3,57 \mathrm{~b} \mathrm{~A}$ & $0,00 \mathrm{~b} \mathrm{~A}$ \\
\hline \multirow{5}{*}{$\begin{array}{c}\mathrm{P} \\
\mathrm{kg} \mathrm{ha}^{-1}\end{array}$} & $0 \%$ & 0,0012 a $\mathrm{A}$ & 0,0011 a $\mathrm{A}$ & 0,0016 a $\mathrm{A}$ \\
\hline & $25 \%$ & $0,0004 \mathrm{ab} \mathrm{A}$ & $0,0002 \mathrm{~b} \mathrm{~A}$ & 0,0003 b A \\
\hline & $50 \%$ & 0,0002 b A & $0,0000 \mathrm{~b} \mathrm{~A}$ & $0,00006 \mathrm{~b} \mathrm{~A}$ \\
\hline & $75 \%$ & $0,00007 \mathrm{~b} \mathrm{~A}$ & $0,00001 \mathrm{~b} \mathrm{~A}$ & $0,00002 \mathrm{~b} \mathrm{~A}$ \\
\hline & $100 \%$ & $0,0000 \mathrm{~b} \mathrm{~A}$ & $0,0000 \mathrm{~b} \mathrm{~A}$ & $0,00001 \mathrm{~b} \mathrm{~A}$ \\
\hline \multirow{5}{*}{$\begin{array}{c}\mathrm{K} \\
\mathrm{kg} \mathrm{ha}^{-1}\end{array}$} & $0 \%$ & 0,0011 a $\mathrm{A}$ & 0,0028 a $\mathrm{A}$ & 0,0018 a $\mathrm{A}$ \\
\hline & $25 \%$ & 0,0004 a A & 0,0014 b A & 0,0007 b A \\
\hline & $50 \%$ & 0,0002 a $\mathrm{A}$ & $0,0001 \mathrm{c} \mathrm{A}$ & $0,00006 \mathrm{~b} \mathrm{~A}$ \\
\hline & $75 \%$ & 0,0000 a $\mathrm{A}$ & 0,0000 с A & $0,00003 \mathrm{~b} \mathrm{~A}$ \\
\hline & $100 \%$ & 0,0000 a A & 0,0000 с A & $0,00001 \mathrm{~b} \mathrm{~A}$ \\
\hline \multirow{5}{*}{$\begin{array}{l}\text { Cálcio } \\
\mathrm{kg} \mathrm{ha}^{-1}\end{array}$} & $0 \%$ & 0,0130 a $\mathrm{A}$ & 0,01656 a $\mathrm{A}$ & 0,0203 a $\mathrm{A}$ \\
\hline & $25 \%$ & 0,0046 b A & $0,0088 \mathrm{a}$ b A & $0,0000 \mathrm{~b} \mathrm{~A}$ \\
\hline & $50 \%$ & $0,0000 \mathrm{~b} \mathrm{~A}$ & $0,0000 \mathrm{~b} \mathrm{~A}$ & 0,0002 b A \\
\hline & $75 \%$ & $0,00008 \mathrm{~b} \mathrm{~A}$ & $0,0000 \mathrm{~b} \mathrm{~A}$ & $0,0001 \mathrm{~b} \mathrm{~A}$ \\
\hline & $100 \%$ & $0,0000 \mathrm{~b} \mathrm{~A}$ & $0,0000 \mathrm{~b} \mathrm{~A}$ & $0,00009 \mathrm{~b} \mathrm{~A}$ \\
\hline \multirow{5}{*}{$\underset{\mathrm{kg} \mathrm{ha}^{-1}}{\mathrm{Mg}}$} & $0 \%$ & $0,0037 \mathrm{a} A$ & 0,0045 a A & 0,0059 a $\mathrm{A}$ \\
\hline & $25 \%$ & 0,0012 b A & $0,0025 \mathrm{~b} \mathrm{~A}$ & $0,0000 \mathrm{~b} \mathrm{~A}$ \\
\hline & $50 \%$ & $0,0000 \mathrm{~b} \mathrm{~A}$ & $0,0000 \mathrm{~b} \mathrm{~A}$ & $0,00006 \mathrm{~b} \mathrm{~A}$ \\
\hline & $75 \%$ & $0,00005 \mathrm{~b} \mathrm{~A}$ & $0,0000 \mathrm{~b} \mathrm{~A}$ & $0,00003 \mathrm{~b} \mathrm{~A}$ \\
\hline & $100 \%$ & $0,0000 \mathrm{~b} \mathrm{~A}$ & $0,0000 \mathrm{~b} \mathrm{~A}$ & $0,00002 \mathrm{~b} \mathrm{~A}$ \\
\hline
\end{tabular}

Médias seguidas de mesma letra minúscula na coluna e maiúscula na linha não diferem estatisticamente, pelo teste de Duncan $(\mathrm{p}<0,05)$, para tipos de cobertura e superfície geomórfica.

O potássio (K) apresentou comportamento diferente daquele do fósforo (Tabela 6). As diferenças foram somente nas posições de encosta e topo. Mas, os valores também foram decrescendo conforme aumentou o percentual da cobertura do solo com palha-de-cana de açúcar. BERTOL et al. (2010) explicam que essa perda decrescente se dá conforme a perda de solo também decresce, como está mostrado na Tabela 6 deste trabalho, já que a concentração desses elementos é maior no solo do que na água. BERTOL et al. (2007) tiveram resultados semelhantes ao estudarem perdas de fósforo e potássio por erosão hídrica em inceptsol.

$\mathrm{O}$ cálcio $(\mathrm{Ca})$ e o magnésio $(\mathrm{Mg})$ tiveram resultado semelhantes conforme a Tabela 6 . Os valores tiveram diferença estatística significativa $(\mathrm{P}<0,05)$. Tanto o $\mathrm{Ca}$ como o $\mathrm{Mg}$ apresentaram perdas nas coberturas de $0,0 \%$ e $25 \%$. Não houve perdas nas coberturas de $50 \%, 75 \%$ e $100 \%$, em todas as superfícies da vertente. Não ocorreu diferença estatística significativa entre as superfícies 
de topo, encosta e sopé. Os valores são 0,013 no topo, 0,0165 na encosta e 0,0203 $\mathrm{Kg} \mathrm{ha}^{-1}$ no sopé, para $\mathrm{Ca}$, no percentual de cobertura de $0,0 \%$. Os valores de $\mathrm{Mg}$ são 0,0037; 0,0045 e 0,0059 Kg ha para as referidas superfícies, também na cobertura de 0,0\%. Essas diferenças de perdas podem ter ocorrido em função da declividade de cada superfície. Comportamento semelhante foi encontrado por BERTOL et al. (2010) ao verificarem valores diferentes ao pesquisarem sistemas de manejo de solo e perdas de nutrientes e matéria orgânica por erosão, em função da declividade.

Os resultados apresentados são concordantes com CASTRO et al. (1986), BEZERRA \& CANTALICE (2006) e MARTINS FILHO et al. (2009), que observaram que as perdas de matéria orgânica e nutrientes têm significativa correlação com as perdas de solo.

\section{CONCLUSÕES}

As concentrações médias de matéria orgânica e nutrientes no sedimento erodido foram significativamente reduzidas quando o solo apresentava cobertura por palha de cana-de-açúcar de $75 \%$ e $100 \%\left(10 \mathrm{tha}^{-1}\right)$.

Enriquecimento do sedimento por matéria orgânica e nutrientes ocorreu quando a cobertura por palha de cana-de-açúcar sobre a superfície do solo era igual ou inferior a $50 \%$.

Independentemente da posição na vertente,seja topo, encosta ou sopé, as perdas de solo foram reduzidas quando aumentou a quantidade de palha de cana-de-açúcar utilizada na cobertura do solo.

\section{REFERÊNCIAS}

BAKKER, M.M.; GOVERS, G.; ROUNSEVELL, M.D.A. The crop productivity- erosion relationship: an analysis based on experimental work. Catena, Amsterdam, v.57, v.1, p.55-76, 2005.

BERTOL, I.; ENGEL, F.L.; MAFRA, A.L.; BERTOL, O.B.; RITTER, S.R. Phosphorus, potassium and organic carbon concentrations in runoff water and sediments under different soil tillage systems during soybean growth. Soil Tillage Research, Amsterdam,v.94, p.142-150, 2007.

BERTOL, I.; VÁZQUEZ, E.V.; GONZÁLEZ, A. P.; COGO, N. P.; LUCIANO, R. V.; FABIAN, E.L. Sedimentos transportados pela enxurrada em eventos de erosão hídrica em um Nitossolo Háplico. Revista Brasileira de Ciência do Solo, Viçosa-MG, v.34 n.1, jan/fev. 2010.

BERTOL, I.; ZOLDAN JR., W.A.; FABIAN, E.L.; ZAVASCHI, E.; PEGORARO, R.; PAZ GONZÁLEZ, A. Efeito de uma escarificação e da erosividade de chuvas sobre algumas variáveis de valores de erosão hídrica em sistemas de manejo de um Nitossolo Háplico. Revista Brasileira de Ciência do Solo, Viçosa-MG, v.32, p.747-757, 2008.

BEZERRA, S.A.; CANTALICE, J.R.B. Erosão entre sulcos em diferentes condições de cobertura do solo sob cultivo da cana-de-açúcar. Revista Brasileira de Ciência do Solo, Viçosa-MG, v.30, n.3, p.565-573, 2006.

CANTALICE, J.R.B.; BEZERRA, S.A.; OLIVEIRA, O.F.; MELO, R.O. Hidráulica e taxas de erosão em entressulcos sob diferentes declividades e doses de cobertura morta. Caatinga, Mossoró, v.22, n.2, p.68-74, 2009.

CASSOL, E.A.; LIMA, V.S.de. Erosão em entressulcos sob diferentes tipos de preparo e manejo do solo. Pesquisa Agropecuaria Brasileira, Brasília, v.38, n.1, p.117-124, 2003.

CASTRO, O.M.; LOMBARDI NETO, F.; QUAGGIO, J. A.; MARIA, I.C.; VIEIRA, S.R.; DECHEN, S.C.F. Perdas por erosão de nutrientes vegetais na sucessão soja/trigo em diferentes sistemas de manejo. Revista Brasileira de Ciência do Solo, Viçosa-MG, v.10, n.3, p.293-297, 1986.

EMBRAPA. EMPRESA BRASILEIRA DE PESQUISA AGROPERCUÁRIA. Centro Nacional de Pesquisa de Solos. Sistema brasileiro de classificação de solos. Brasília: Embrapa Produção de informação; Rio de Janeiro: Embrapa Solos, 2006. 412 p. 
EMBRAPA. EMPRESA BASILEIRA DE PESQUISA AGROPERCUÁRIA. Serviço Nacional de levantamento e conservação de solos. Manual de métodos de análise do solo. Rio de Janeiro, 1979. Não paginado.

FREITAS, J.F.; CANTALICE, J.R.B.; BEZERRA, S.A.; SILVA, M.D.R.; LIMA, P.A. Erosão em entressulcos sob caatinga e culturas agrícolas. Revista Brasileira de Ciência do Solo, Viçosa, v.32, n.4, p.1.743-1.751, 2008.

IZIDORIO, R.; MARTINS FILHO, M. V.; MARQUES JÚNIOR, J.; SOUZA, Z.M.; PEREIRA, G.T. Perdas de nutrientes por erosão e sua distribuição espacial em área sob cana-de-açúcar. Engenharia Agrícola, Jaboticabal, v.25, n.3, p.660-670, 2005.

MARQUES JÚNIOR, J. Distribuição e atributos dos solos em relação à forma e evolução de uma vertente em Monte Alto-SP. 1995. 226 f. Tese (Doutorado em Solos e Nutrição de Plantas) - Escola Superior de Agricultura “Luiz de Queiroz”, Universidade de São Paulo, Piracicaba, 1995.

MARTINS FILHOS, M.V.; LICCIOTI, T.T.; PEREIRA, G.T.; MARQUES JÚNIOR, J.;

SANCHEZ, R.B. Perdas de solo e nutrientes por erosão num Argissolo com resíduos vegetais de cana-de-açúcar. Engenharia Agrícola, Jaboticabal, v.29, n.1, p.8-18, 2009.

RAIJ, B. V.; ANDRADE, J.C.; CANTARELLA, H.; QUAGGIO, J.A. Análise química para avaliação da fertilidade de solos tropicais. Campinas: Secretaria de Agricultura e Abastecimento, APTA e IAC, 2001. 284p.

SCHICK, J.; BERTOL, I.; BALBINOT JR., A.A. BATISTELA, O. Erosão hídrica em Cambissolo Húmico alumínico submetido a diferentes sistemas de preparo e cultivo do solo: II. Perdas de nutrientes e carbono orgânico. Revista Brasileira de Ciência do Solo, Viçosa-MG, v.24, p.437-447, 2000 . 